\title{
SEDATALOG: A SET EXTENSION OF DATALOG
}

\author{
Qing Zhou \\ The Software Institute of Zhongshan University \\ Guangzhou, Guangdong 510275, P.R.China \\ Inszq@zsulink.zsu.edu.cn \\ Ligong Long \\ The Software Institute of Zhongshan University \\ Guangzhou, Guangdong 510275, P.R.China \\ longligong@tom.com
}

\begin{abstract}
In this paper we propose an extension, SEDatalog, of Datalog so that sets can be naturally constructed in logic programming. In $S E D$ atalog, sets can be defined by statements so it has a strong capability in creating sets. Three deductive rules are also introduced in this paper, which make $S E D$ atalog strong in deductions and programming even when sets are involved in deductions. The syntactical description and the semantical interpretation of SEDatalog are comprehensively discussed in detail. The soundness and completeness theorem of $S E D a t a l o g$ is proved, which provides a solid foundation of $S E D$ atalog.
\end{abstract}

Keywords: The order of a set, The order of predicate, $n$-th order set

\section{Introduction}

In this paper we propose an extension, SEDatalog, of Datalog so that sets can be constructed in logic programming. As the extension is entirely based on what is common in every logic programming language, the extension could apply to Prolog and other logic programming languages almost without any modification.

In $S E$ Datalog, we can define a set $A$ such that $A=\{x: p(x)\}$ for every formula. By defining sets by statements we can not only construct finite sets but also infinite sets or more complicated sets such as a set of sets with certain properties. Also this is the way we define sets when we are working in mathematics, or in other areas. This definition, if without proper restrictions, would involve confusions among set construction levels and would lead to Russell's paradox. To avoid this, we restrict the elements to be contained in a set to only 
those which already exist, thus helping achieve clear indication of a set hierarchy to be constructed in SEDatalog. As hierarchies constructed this way are in line with the underlying principles of the axiomatic set theory i.e. $Z F$, avoidance of such paradoxes as "the set of all sets" or "a set containing itself" can be assured.

For this purpose we need an "order" for every set which indicates the level in which the set is constructed. Then statements in Datalog can be used to define sets. We consider that these sets are of the first order. Using these sets and individuals in Datalog, which are of order 0, we can construct a group of statements, which are not in Datalog in general, by which second order sets can be defined. Continuing by this way, we may have all the sets constructed. To ensure avoidance of any possible confusion in the construction, we have to give an order to every statement in $S E D$ atalog, too. This means that every predicate in $S E D$ atalog can only allow those sets with order less than a constant integer (the order of the predicate) as its variables. So every predicate in SEDatalog is a partial predicate, i.e. its domain can not contain any set which has the order larger than or equal to the order of the predicate. This takes care of the problem mentioned in the last paragraph.

\section{The Syntactical Description Of SEDatalog}

The alphabet of $S E D$ atalog consists of variables, constants, predicates and connectives. Each constant, variable and predicate is assigned an unique integer to it. A $n$-th order term is a constant or variable which has been assigned $n$ to it. The order of a predicate is $n$, if it is assigned $n$. We will use $o(t)$ to indicate the order of $t$ if $t$ is a variable, a constant or a predicate.

DEFINITION 1 Formulas and their orders are defined as follows:

(1) If $p$ is a $k$-order $n$-ary predicate symbol, and for every $i, 1 \leq i \leq n$, $t[i]$ is a term with $o(t[i])<k$, then $p(t[1], \ldots, t[n])$ is an atom formula and $o(p(t[1], \ldots, t[n]))=k$;

(2) If $t[1]$ and $t[2]$ are terms, and $o(t[1])<o(t[2])$, then $t[1] \in t[2]$ is an atom formula and $o(t[1] \in t[2])=o(t[2])+1$;

(3) If $t[1]$ and $t[2]$ are terms, and $0<o(t[1]) \leq o(t[2])$, then $t[1] \subseteq t[2]$ is an atom formula and $o(t[1] \subseteq t[2])=o(t[2])+1$;

(4) If $t[1]$ and $t[2]$ are terms, and $o(t[1])=o(t[2])$, then $t[1]=t[2]$ is an atom formula and $o(t[1]=t[2])=o(t[2])+1$.

(5) If $F, G$ are formulas, then $F \wedge G, F \vee G$ are formulas and $o(F \wedge G)=$ $\max (\{o(F), o(G)\}), o(F \vee G)=\max (\{o(F), o(G)\})$.

An atom with no free variables is called a ground atom. A closed formula is a formula with no free variables. An atom is called a literal in SEDatalog.

Remark In the above definition, $F(x) \vee G(x)$ might not be well defined from Definition 1 for some $x$ with order $o(F(x)) \leq o(x)<o(G(x))$ when 
$o(F(x))<o(G(x))$. To let the definition make sense, we consider it as $F^{\prime}(x) \vee$ $G(x)$, where $F^{\prime}(x) \leftrightarrow F(x)$ and $o\left(F^{\prime}(x)\right)=o(G(x))$, i.e. the domain of $F^{\prime}(x)$ is extended to all terms with the order up to $o(G(x))$, although $F^{\prime}(x) \leftrightarrow$ $F(x)$. The same treatment is made for $F(x) \wedge G(x)$.

DEFINITION 2 For each formula $F(x)$ with only one free variable $x$, the set defined by $F(x)$ is a constant $C_{F(x)} \in C_{n}$ with $n=o(F(x))$ such that for all $t, t \in C_{F(x)}$ if and only if $F(t)$.

We often write $C_{F(x)}=\{t: F(t)\}$ to indicate the definition of $C_{F(x)}$.

Directly from our definitions, it is not hard to see that for every formula $F(x), o\left(C_{F(x)}\right)$ is a finite integer.

Then we turn to the deduction rules of SEDatalog. As an extension of Datalog, we first extend the deduction rule of Datalog to be used on statements which contain sets as their variables. We call it "the ordinary rule". In addition to this ordinary rule, we add two more deduction rules, "the universal rule" and "the existential rule".

DeFinition 3 A rule of $S E D$ atalog is of the form $H:-A_{1}, \ldots, A_{n}$ where $n \geq 0$. The left hand side of: - is a literal, called the head of the rule, while the right hand side is a conjunction of literals, called the body of the rule .

A fact is a special rule, whose head is a ground literal and whose body is empty.

For convenience, we use the notation $\operatorname{vars}(T)$ to indicate all variables occurring in $T$, where $T$ is a term or a formula. Then vars is a mapping from the set of terms and formulas to the power set of $V a r$. We use $H(y)$ to represent a literal with variable $y$ and $A(x)$ to represent a literal with variable $x$. Now we give the following three rules, which will be called safe. They are the basic deductive rules in SEDatalog:

(1) Ordinary rule is of the form

$$
H:-o A_{1}, \ldots, A_{n}
$$

where $n>0, \operatorname{var} s(H) \subseteq \operatorname{vars}\left(A_{1} \wedge \ldots \wedge A_{n}\right)$ and $o(H)=o\left(A_{1} \wedge \ldots \wedge A_{n}\right)$.

The informal semantics of this rule is to mean that "for every assignment to each variable, if $A_{1}, \ldots, A_{n}$ are true, then $H$ is true"

(2) Universal rule is of the form

$$
H(y):{ }_{U} A_{1}(x), \ldots, A_{m}(x), A_{m+1}, \ldots, A_{n}
$$

where $\operatorname{vars}(H(y))-\{y\} \subseteq \operatorname{vars}\left(A_{1}(x) \wedge \ldots \wedge A_{m}(x) \wedge A_{m+1} \wedge \ldots \wedge A_{n}\right)-\{x\}$, $y \neq x, o(H(y))>o\left(A_{1}(x) \wedge \ldots \wedge A_{m}(x) \wedge A_{m+1} \wedge \ldots \wedge A_{n}\right)$ and $y \subseteq\{x:$ $\left.A_{1}(x) \wedge \ldots \wedge A_{m}(x) \wedge A_{m+1} \wedge \ldots \wedge A_{n}\right\}$. 
The informal semantics of this rule is to mean that "if every element $x$ in $y$ has properties $A_{1}(x), \ldots, A_{m}(x)$, then $y$ has the property $H$ ".

In this case, $y$ is called of the universal property $H$.

(3) Existential rule is of the form

$$
\begin{aligned}
& H(y):-{ }_{E} \\
& \quad A_{1}\left(x_{1}, \ldots, x_{k}\right), \ldots, A_{m}\left(x_{1}, \ldots, x_{k}\right), A_{m+1}, \ldots, A_{n}
\end{aligned}
$$

where $\operatorname{vars}(H(y))-\{y\} \subseteq \operatorname{vars}\left(A_{1}\left(x_{1}, \ldots, x_{k}\right) \wedge \ldots \wedge A_{m}\left(x_{1}, \ldots, x_{k}\right) \wedge\right.$ $\left.A_{m+1} \wedge \ldots \wedge A_{n}\right)-\left\{x_{1}, \ldots, x_{k}\right\}, y \neq x_{1}, \ldots, y \neq x_{k}, o(H(y))>o\left(A_{1}\left(x_{1}, \ldots\right.\right.$ ,$\left.\left.x_{k}\right) \wedge \ldots \wedge A_{m}\left(x_{1}, \ldots, x_{k}\right) \wedge A_{m+1} \wedge \ldots \wedge A_{n}\right)$ and $x_{1} \in y, \ldots, x_{k} \in y$.

The informal semantics of this rule is to mean that "if some elements $x_{1}, \ldots$ ,$x_{k}$ in $y$ have properties $A_{1}\left(x_{1}, \ldots, x_{k}\right), \ldots, A_{m}\left(x_{1}, \ldots, x_{k}\right)$, then $y$ has the property $H^{\prime \prime}$.

In this case, $y$ is called of the existential property $H$.

\section{DEFINITION 4 A SEDatalog program is a finite sequence of rules.}

DEFINITION 5 A substitution $\theta$ is a finite set of the form $\left\{x_{1} / t_{1}, \ldots, x_{n} / t_{n}\right\}$, where $x_{1}, \ldots, x_{n}$ are distinct variables and each $t_{i}$ is a term such that $x_{i} \neq t_{i}$, and $o\left(t_{i}\right) \leq o\left(x_{i}\right)$.

The set of variables $\left\{x_{1}, \ldots, x_{n}\right\}$ is called the domain of $\theta$.

If $T$ is a term, a literal or a rule then $T \theta$ denotes the corresponding item obtained from $T$ by simultaneously replacing each $x_{i}$ that occurs in $T$ by the corresponding term $t_{i}$, if $x_{i} / t_{i}$ is an element of $\theta$.

If each $t_{i}$ is ground, then $\theta$ is a ground substitution.

\section{The Semantical Interpretations of SEDatalog}

Let $M_{0}=\left\langle\mathcal{V}, \mathcal{P}_{0}, \mathcal{T}_{0}\right\rangle$ be an interpretation of Datalog, where $\mathcal{V}$ is the universe of $M_{0} ; \mathcal{P}_{0}$ is the set of the interpretations of predicate symbols of Datalog; $\mathcal{T}_{0}$ is the set of interpretations of those ground atoms of Datalog which are interpreted as true; respectively. We define $\mathcal{U}_{0}=\mathcal{V}$, and $\mathcal{U}_{n}=$ $\mathcal{U}_{n-1} \cup \wp\left(\mathcal{U}_{n-1}\right)$ where $\wp\left(\mathcal{U}_{n-1}\right)$ is the power set of $\mathcal{U}_{n-1}$, i.e. $\wp\left(\mathcal{U}_{n-1}\right)=$ $\left\{A: A \subseteq \mathcal{U}_{n-1}\right\}$. Then we give the full description of the interpretation of $S E$ Datalog as follows:

An interpretation $M$ of $S E D$ atalog is a tuple: $M=\langle\mathcal{U}, \mathcal{P}, \mathcal{T}\rangle$, here $\mathcal{U}=\bigcup_{k=0}^{\infty} \mathcal{U}_{k}$ is the universe of $M ; \mathcal{P}$ is the set of the interpretations of predicate symbols; $\mathcal{T}$ is the set of interpretations of those ground literals which are interpreted as true, respectively, such that:

(1) Each $n$-ary predicate symbol $q_{\langle k\rangle}$ is interpreted as a predicate $q_{M} \in \mathcal{P}$, i.e. $q_{M} \subseteq \mathcal{U}_{k}^{n}$, and $q_{\langle 1\rangle}$ is interpreted as a predicate $q_{M} \in \mathcal{P}_{0}$;

Especially, $\epsilon_{\langle m\rangle}, \subseteq_{\langle m\rangle}$ and $=\langle m\rangle$ are interpreted as the usual meanings. 
(2) Each constant $c$ in $C_{n}(n>0)$ is interpreted as an object (set) $M(c)$ of $\mathcal{U}_{n}$; and each constant $c$ in $C_{0}$ is interpreted as same as in $M_{0}$, i.e. an object (individual) $M(c)$ of $\mathcal{U}_{0}$;

(3) A ground atom $q(t[1], \ldots, t[n])$ is interpreted as $M(q(t[1], \ldots, t[n])) \Longleftrightarrow$ $q_{M}(M(t[1]), \ldots, M(t[n]))$, for more complicated formulas such as $F(x) \vee$ $G(x)$ and $F(x) \wedge G(x)$, their truth values are interpreted as usual;

(4) Now we define $\mathcal{T}$ as follows:

$\mathcal{T}_{0}$ is as same as the $\mathcal{T}_{0}$ in $M_{0}$;

$\mathcal{T}_{k} \subseteq \mathcal{T}_{k-1} \cup\left\{q_{M}(M(t[1]), \ldots, M(t[n])): q_{<k>}(t[1], \ldots, t[n])\right.$ is a ground literal $\}$ which satisfies $\mathcal{T}_{k-1} \subset \mathcal{T}_{k}$ and:

i) $\left.M\left(c \in C_{A(x)}\right)\right) \in \mathcal{T}_{k}$ if and only if $M(A(c)) \in \mathcal{T}_{k-1}$.

ii) $M\left(C_{F(x)} \subseteq C_{G(x)}\right) \in \mathcal{T}_{k}$ if and only if for all $x \in \mathcal{U}, M(F)(x) \in \mathcal{T}$ implies that $M(G)(x) \in \mathcal{T}_{k}$.

and finally, let $\mathcal{T}=\bigcup_{k=1}^{\infty} \mathcal{T}_{k}$.

With the interpretation of $S E D$ atalog described above, our next job is to give the description of the model of a $S E D$ atalog program:

Let $P$ be a program. An interpretation $M=\langle\mathcal{U}, \mathcal{P}, \mathcal{T}\rangle$ is a model of $P$ if and only if

(1) If $A$ is a fact in $P$, then $M(A) \in \mathcal{T}$;

(2) If $r: H:-O A_{1}, \ldots, A_{n}$ is an ordinary rule in $P$, then for each ground and legal substitution $\theta$ with $\operatorname{domain}(\theta) \supseteq \operatorname{vars}(r)$, if $M\left(A_{1} \theta\right) \in \mathcal{T}, \ldots$, $M\left(A_{n} \theta\right) \in \mathcal{T}$, then $M(H \theta) \in \mathcal{T}$;

(3) If $r: H(y):-U A_{1}(x), \ldots, A_{m}(x), A_{m+1}, \ldots, A_{n}$ is a universal rule in $P$, then for each ground and legal substitution $\theta$ with $\operatorname{domain}(\theta)=\operatorname{vars}(r)-$ $\{x\}$, if $M\left(A_{m+1} \theta\right) \in \mathcal{T}, \ldots, M\left(A_{n} \theta\right) \in \mathcal{T}$, and $M\left(y \theta \subseteq C_{A_{1}(x) \theta}\right) \in \mathcal{T}, \ldots$, $M\left(y \theta \subseteq C_{A_{m}(x) \theta}\right) \in \mathcal{T}$, then $M(H(y) \theta) \in \mathcal{T}$, here $M\left(y \theta \subseteq C_{A_{i}(x) \theta}\right) \in \mathcal{T}$, $1 \leq i \leq m$

(4) If $r: H(y):-{ }_{E} A_{1}\left(x_{1}, \ldots, x_{k}\right), \ldots, A_{m}\left(x_{1}, \ldots, x_{k}\right), A_{m+1}, \ldots, A_{n}$ is a existential rule in $P$, then for each ground and legal substitution $\theta$ with $\operatorname{domain}(\theta)=\operatorname{vars}(r)$, if $M\left(A_{1}\left(x_{1}, \ldots, x_{k}\right) \theta\right) \in \mathcal{T}, \ldots, M\left(A_{m}\left(x_{1}, \ldots\right.\right.$ ,$\left.\left.x_{k}\right) \theta\right) \in \mathcal{T}, M\left(A_{m+1} \theta\right) \in \mathcal{T}, \ldots, M\left(A_{n} \theta\right) \in \mathcal{T}$, and $M\left(\left(x_{1} \in y\right) \theta\right) \in \mathcal{T}, \ldots$ , $M\left(\left(x_{k} \in y\right) \theta\right) \in \mathcal{T}$ then $M(H(y) \theta) \in \mathcal{T}$.

Definition 6 Let $A$ be a ground literal. An interpretation $M=\langle\mathcal{U}, \mathcal{P}, \mathcal{T}\rangle$ is a model of $A$ if and only if $M(A) \in \mathcal{T}$.

Now we are going to discuss the soundness and completeness theorem of $S E D a t a l o g$. Before that let us introduce some related notions first.

DEFINITION 7 A ground literal $A$ is a consequence of a SEDatalog program $P$ (denoted by $P \models A$ ) if and only if each model $M$ of $P$ is also a model of $A$. 
DEFINITION $8 A$ ground literal $A$ is inferred from a $S E D$ Datalog program $P$ (denoted by $P \vdash A$ ) is defined as follows:

(1) If $A=H$ and $H$ is a fact in $P$, then $P \vdash A$;

(2) If there exists an ordinary rule $r: H:-O A_{1}, \ldots, A_{n}$ in $P$ and a ground and legal substitution $\theta$, where domain $(\theta)=\operatorname{vars}(r)$, such that $A=H \theta$ and $P \vdash A_{1} \theta, \ldots, P \vdash A_{n} \theta$, then $P \vdash A$;

(3) If there exists a universal rule $r: H(y):-{ }_{U} A_{1}(x), \ldots, A_{m}(x), A_{m+1}, \ldots$ , $A_{n}$ in $P$ and a ground and legal substitution $\theta$, where domain $(\theta)=\operatorname{vars}(r)-$ $\{x\}$, such that $A=H(y) \theta$ and $P \vdash A_{m+1} \theta, \ldots, P \vdash A_{n} \theta$, and $P \vdash y \theta \subseteq$ $C_{A_{1}(x) \theta}, \ldots, P \vdash y \theta \subseteq C_{A_{m}(x) \theta}$, then $P \vdash A$, here $P \vdash y \theta \subseteq C_{A_{i}(x) \theta}$, $1 \leq i \leq m$;

(4) If there exists an existential rule $r: H(y):-{ }_{E} A_{1}\left(x_{1}, \ldots, x_{k}\right), \ldots$ , $A_{m}\left(x_{1}, \ldots, x_{k}\right), A_{m+1}, \ldots, A_{n}$ in $P$ and a ground and legal substitution $\theta$, where domain $(\theta)=\operatorname{vars}(r)$, such that $A=H(y) \theta$ and $P \vdash A_{1}\left(x_{1}, \ldots\right.$ ,$\left.x_{k}\right) \theta, \ldots, P \vdash A_{m}\left(x_{1}, \ldots, x_{k}\right) \theta, P \vdash A_{m+1} \theta, \ldots, P \vdash A_{n} \theta, P \vdash x_{1} \theta \in y \theta$, ..., $P \vdash x_{k} \theta \in y \theta$, then $P \vdash A$.

Let infer $(P)=\{A: P \vdash A\}$ and $\operatorname{cons}(P)=\{A: P \models A\}$. It is easy to show that, $\operatorname{cons}(P)=\bigcap_{M}\{A: M=\langle\mathcal{U}, \mathcal{P}, \mathcal{T}\rangle$ is a model of $P$ and $M(A) \in$ $\mathcal{T}$.\}. Then we can prove The Soundness and Completeness Theorem:

THEOREM 9 infer $(P)=\operatorname{cons}(P)$.

\section{References}

Abiteboul, S.,Grumbach, S.,: COL: A Logic-based Language for Complex Objects, ACM TODS, 16(1), pp.1-30, 1991.

Ceri, S., Gottlob, G., Tanca, L.,: Logic Programming and Databases, Springer Verlag, 1990.

Chimenti, D., Gamboa, R., Krishnamurthy, R., Naqvi, S., Tsur, S.,Zaniolo, C.,; The LDL System Prototype, IEEE Transactions on Knowledge and Data Engineering, 2(1), pp.76-90, 1990.

Dovier, A., Omodeo, E. G., Pontelli, E., Rossi, G.,: $\{\log \}$ : A Language for Programming in Logic with Finite Sets, J. of Logic Programming, 28(1), pp.1-44, 1996.

Jana, D., Semantics of Subset-Logic Languages, Ph.D. Dissertation, Department of Computer Science, SUNY-Buffalo, 1994.

Jayaraman, B.,: The SuRE Programming Framework, TR 91-011, Department of Computer Science, SUNY-Buffalo, August 1991.

Jayaraman, B., Jana, D.,: Set Constructors, Finite Sets, and Logical Semantics, J. of Logic Programming, 38, pp.55-77, 1999.

Kuper, G. M.,: Logic Programming with Sets, J. of Computer and System Science, 41(1), pp.4464, 1990.

Liu, M.,: Relationlog: A Typed Extension to Datalog with Sets and Tuples, J. of Logic Programming, 36, pp.271-299, 1998.

Lloyd, J. W.,: Foundations of Logic Programming, Springer Verlag, 1987.

Moon, K.,: Implementation of Subset Logic Languages, Ph.D. Dissertation, Department of Computer Science, SUNY-Buffalo, February 1995.

Osorio, M.,: Semantics of Logic Programs with Sets, Ph.D. Dissertation, Department of Computer Science, SUNY-Buffalo, 1995. 